\title{
Electrically tunable, ultranarrowband, circular-polarization rejection filters with electro-optic structurally chiral materials
}

\begin{abstract}
Akhlesh Lakhtakia
akhlesh@psu.edu

CATMAS - Computational \& Theoretical Materials Sciences Group, Department of Engineering Science \& Mechanics, Pennsylvania State University, University Park, PA 16802-6812, USA

The transmittance spectrum of a slab of an electro-optic structurally chiral material (SCM) that is helicoidally non homogeneous in the thickness direction and is endowed with a central $90^{\circ}$-twist defect, shows evidence of an ultranarrowband spectral hole when a sufficiently high dc electric field is applied between the entry and the exit planes and the incident light is circularly polarized in opposition to the structural handedness of the SCM. This spectral hole migrates on the wavelength axis as the applied dc electric field is altered in magnitude, thereby suggesting the possible use of a centrally defective, electro-optic SCM slab as an electrically tunable, circular-polarization rejection filter with ultra narrow bandwidth. [DOI: 10.2971/jeos.2006.06006]
\end{abstract}

Keywords: Circular Bragg phenomenon, circular polarization, electro-optic filter, phase defect, Pockels effect, rejection filter, spectral hole, structurally chiral material, tunable filter

\section{I n trod uct io n}

Volume gratings are generally made of isotropic dielectric materials. The relative permittivity of a volume grating varies periodically in the thickness direction from the entry plane to the exit plane [1, 2], and therefore it exhibits the Bragg phenomenon: the existence of a high-reflectance wavelengthregime, provided the grating thickness spans a large number of relative-permittivity periods. Haus and Shank [3] proposed the insertion of a phase defect half-way along a volume grating, in order to create a spectral hole in the reflection spectrum - a transmission feature in the center of the Bragg regime which is useful for narrow-bandpass filtering [4].

On normal illumination, the Bragg phenomenon is insensitive to the polarization state of the incident light, because the volume gratings are made of isotropic materials. However, structurally chiral materials (SCMs), being anisotropic and periodically non homogeneous, exhibit a circular-polarizationsensitive Bragg phenomenon even on normal illumination. SCMs are exemplified by cholesteric liquid crystals $[5,6]$ as well as chiral sculptured thin films [7]. As the relative permittivity matrix of a SCM varies helicoidally in the thickness direction, the high-reflectance characteristic of the Bragg regime is observed only when the incident light is circularly polarized $(\mathrm{CP})$ in such a way that its handedness matches the structural handedness of the material; but incident CP light of the other handedness is mostly transmitted. This distinction suggests that the term circular Bragg phenomenon (CBP) be used for the most important optical characteristic of SCMs.

The introduction of a phase defect half-way inside a SCM slab also gives rise to a spectral hole - but only when the CP state of the incident light matches the structural handedness $[8,9]$. This spectral hole in the co-handed reflectance spectrum of a
SCM slab can be exploited for narrow-bandpass filtering [10] as well as for sensing fluid infiltrants [11].

The CP-sensitivity of structurally chiral spectral-hole filters can not, of course, be exhibited by isotropic spectral-hole filters. Even more interestingly, as the SCM slab with a central phase defect becomes thicker, but with its period fixed, the spectral hole in the co-handed reflectance spectrum diminishes steadily and eventually vanishes. Simultaneously, a spectral hole appears, grows, and eventually matures in the transmittance spectrum for incident light of the other CP state. The bandwidth of the second spectral hole is a tiny fraction of the bandwidth of the first spectral hole, according to theoretical calculations [12]-[14].

The two spectral holes and the crossover from one to the other have been taken to indicate the arousal of a defect mode. The defect mode has been subjected to several theoretical studies with both exact and approximate analytical techniques [15][19]. The possibility of exploiting the defect mode for emission of narrow band CP light is also very attractive [20, 21]. However, the second spectral hole (in the cross-handed transmission spectrum) has yet not been experimentally realized possibly, because the SCM thickness required is so large that the presence of even small dissipation is ruinous [13, 22].

The key then to realize the ultranarrowband spectral hole in the cross-handed transmission spectrum is to somehow reduce the thickness of the SCM slab with the central phase defect. Such a reduction could be possible if the SCM were to display the Pockels effect [23], which thought was examined and validated for a SCM endowed with a local $\overline{4} 2 \mathrm{~m}$ point group symmetry [24]. That validation necessarily led to the issue of SCMs with other local point group symmetries being more 
suitable than the one with local $\overline{4} 2 m$ point group symmetry, and engendered this more comprehensive report on the possible use of a centrally defective, electro-optic SCM slab as an electrically tunable, CP rejection, ultranarrowband filter.

The plan of this paper is as follows: Section 2 contains a description of the boundary-value problem when a CP plane wave is normally incident on a SCM slab with local point group symmetry of one of 20 types and containing a central phase defect. Although the phase defect can be in several different forms $[25,26]$, here we take it as a $90^{\circ}$-twist defect about the thickness axis. Section 3 contains numerical results that theoretically demonstrate the feasibility of exploiting an electro-optic SCM slab with a central $90^{\circ}$-twist defect as an electrically tunable, $\mathrm{CP}$ rejection filter with ultra narrow bandwidth.

A note about notation: Vectors are denoted in boldface; the Cartesian unit vectors are represented by $\hat{\mathbf{u}}_{x}, \hat{\mathbf{u}}_{y}$, and $\hat{\mathbf{u}}_{z} ;$ symbols for column vectors and matrices are decorated by an over bar; and an $\exp (-i \omega t)$ time-dependence is implicit with $\omega$ as the angular frequency, $t$ as time, and $i=\sqrt{-1}$. The wavenumber and the intrinsic impedance of free space (i.e., vacuum) are denoted by $k_{0}=\omega \sqrt{\epsilon_{0} \mu_{0}}$ and $\eta_{0}=\sqrt{\mu_{0} / \epsilon_{0}}$, respectively, with $\mu_{0}$ and $\epsilon_{0}$ being the permeability and permittivity of free space.

\section{B O U N D A R - V A L U E P R B LEM}

Suppose that an electro-optic SCM slab with a central $90^{\circ}$ twist defect occupies the region $0 \leq z \leq 2 L$, the half spaces $z \leq 0$ and $z \geq 2 L$ being vacuous. The SCM slab has the $z$ axis as its axis of helicoidal non homogeneity, and is subject to a dc electric field $\mathbf{E}^{d c}=E_{z}^{d c} \hat{\mathbf{u}}_{z}$. An arbitrarily polarized plane wave is normally incident on the device from the half space $z \leq 0$. In consequence, a reflected plane wave also exists in the same half space and a transmitted plane wave in the half space $z \geq 2 L$.

The total electric field phasor in the half space $z \leq 0$ is given by

$$
\begin{aligned}
& \mathbf{E}(\mathbf{r})=\left(a_{L} \hat{\mathbf{u}}_{+}+a_{R} \hat{\mathbf{u}}_{-}\right) \exp \left(i k_{0} z\right)+ \\
& \left(r_{L} \hat{\mathbf{u}}_{-}+r_{R} \hat{\mathbf{u}}_{+}\right) \exp \left(-i k_{0} z\right), \quad z \leq 0,
\end{aligned}
$$

where $\mathbf{u}_{ \pm}=\left(\hat{\mathbf{u}}_{x} \pm i \hat{\mathbf{u}}_{y}\right) / \sqrt{2}$. Likewise, the electric field phasor in the half space $z \geq 2 L$ is represented as

$$
\mathbf{E}(\mathbf{r})=\left(t_{L} \hat{\mathbf{u}}_{+}+t_{R} \hat{\mathbf{u}}_{-}\right) \exp \left[i k_{0}(z-2 L)\right], \quad z \geq 2 L .
$$

Here, $a_{L}$ and $a_{R}$ are the known amplitudes of the left- and the right-CP (LCP \& RCP) components of the incident plane wave; $r_{L}$ and $r_{R}$ are the unknown amplitudes of the reflected plane wave components; while $t_{L}$ and $t_{R}$ are the unknown amplitudes of the transmitted plane wave components. The aim in solving the boundary value problem is to determine $r_{L, R}$ and $t_{L, R}$ for known $a_{L}$ and $a_{R}$

\subsection{0ptical Relative Permittivity Matrix of the SCM Slab}

In line with the optical relative permittivity matrix of nonelectro-optic SCM slabs with central $90^{\circ}$-twist defects $[7,10$, 11] that of the electro-optic SCM slab may be stated as follows [24]:

$$
\begin{aligned}
& \bar{\epsilon}^{S C M}(z)=\bar{S}_{z}\left[h \frac{\pi z}{\Omega}+h \psi(z)\right] \cdot \bar{R}_{y}(\chi) \cdot \bar{\epsilon}_{P E} . \\
& \bar{R}_{y}(\chi) \cdot \bar{S}_{z}^{-1}\left[h \frac{\pi z}{\Omega}+h \psi(z)\right], \quad 0<z<2 L .
\end{aligned}
$$

The tilt matrix

$$
\bar{R}_{y}(\chi)=\left(\begin{array}{ccc}
-\sin \chi & 0 & \cos \chi \\
0 & -1 & 0 \\
\cos \chi & 0 & \sin \chi
\end{array}\right)
$$

depends on the angle $\chi \in[0, \pi / 2]$ with respect to the $x$ axis in the $x z$ plane; it contains a rotation about the $y$ axis for generality, thereby letting Eq. (3) mimic the morphologies of both chiral smectic liquid crystals [6] and chiral sculptured thin films [7]. The use of the rotation matrix

$$
\bar{S}_{z}(\zeta)=\left(\begin{array}{ccc}
\cos \zeta & -\sin \zeta & 0 \\
\sin \zeta & \cos \zeta & 0 \\
0 & 0 & 1
\end{array}\right)
$$

in Eq.(3) involves the half-pitch $\Omega$ of the SCM along the $z$ axis. In addition, the handedness parameter $h=1$ for structural right-handedness and $h=-1$ for structural left-handedness. The angle $\psi(z)$ delineates the central $90^{\circ}$-twist defect as

$$
\psi(z)= \begin{cases}0, & 0<z<L \\ \pi / 2, & L<z<2 L .\end{cases}
$$

As the two halves of the SCM slab are supposed to be identical, the ratio $L / \Omega$ is conveniently taken to be an integer.

Correct to the first order in the components of the dc electric field, the approximation

$$
\bar{\epsilon}_{P E} \approx\left(\begin{array}{ccc}
\epsilon_{1}^{(0)}\left(1-\epsilon_{1}^{(0)} S_{1}\right) & -\epsilon_{1}^{(0)} \epsilon_{2}^{(0)} S_{6} & -\epsilon_{1}^{(0)} \epsilon_{3}^{(0)} S_{5} \\
-\epsilon_{2}^{(0)} \epsilon_{1}^{(0)} S_{6} & \epsilon_{2}^{(0)}\left(1-\epsilon_{2}^{(0)} S_{2}\right) & -\epsilon_{2}^{(0)} \epsilon_{3}^{(0)} S_{4} \\
-\epsilon_{3}^{(0)} \epsilon_{1}^{(0)} S_{5} & -\epsilon_{3}^{(0)} \epsilon_{2}^{(0)} S_{4} & \epsilon_{3}^{(0)}\left(1-\epsilon_{3}^{(0)} S_{3}\right)
\end{array}\right),
$$

where $S_{i}=\sum_{K=1}^{3} r_{i K} E_{K}^{d c}$, emerges from the reciprocal of the optical relative permittivity matrix of a homogeneous electrooptic material. This latter matrix is usually reported as [23]

$$
\bar{\epsilon}_{P E}^{-1}=\left(\begin{array}{ccc}
1 / \epsilon_{1}^{(0)}+S_{1} & S_{6} & S_{5} \\
S_{6} & 1 / \epsilon_{2}^{(0)}+S_{2} & S_{4} \\
S_{5} & S_{4} & 1 / \epsilon_{3}^{(0)}+S_{3}
\end{array}\right)
$$

in the principal Cartesian coordinate system (with axes labeled 1, 2, and 3) relevant to the crystallographic structure of a homogeneous material displaying the Pockels effect. Here, $\epsilon_{1,2,3}^{(0)}$ are the three principal relative permittivity scalars in the optical regime, whereas $r_{J K}$ (with $1 \leq J \leq 6$ and $1 \leq K \leq 3$ ) are the electro-optic coefficients in the traditional contracted 
or abbreviated notation for representing symmetric secondorder tensors [23]. Finally,

$$
\left.\begin{array}{l}
E_{1}^{d c}=E_{z}^{d c} \cos \chi \\
E_{2}^{d c}=0 \\
E_{3}^{d c}=E_{z}^{d c} \sin \chi
\end{array}\right\}
$$

are the components of the applied dc electric field.

\subsection{Reflectances and Transmit- tances}

The procedure to obtain the unknown reflection and transmission amplitudes involves the $4 \times 4$ matrix relation $[7,24]$

$$
\bar{f}_{\text {exit }}=\bar{M} \cdot \bar{f}_{\text {entry }},
$$

where the column 4-vectors

$$
\bar{f}_{\text {entry }}=\frac{1}{\sqrt{2}}\left(\begin{array}{c}
\left(r_{L}+r_{R}\right)+\left(a_{L}+a_{R}\right) \\
i\left[-\left(r_{L}-r_{R}\right)+\left(a_{L}-a_{R}\right)\right] \\
-i\left[\left(r_{L}-r_{R}\right)+\left(a_{L}-a_{R}\right)\right] / \eta_{0} \\
-\left[\left(r_{L}+r_{R}\right)-\left(a_{L}+a_{R}\right)\right] / \eta_{0}
\end{array}\right)
$$

and

$$
\bar{f}_{\text {exit }}=\frac{1}{\sqrt{2}}\left(\begin{array}{c}
t_{L}+t_{R} \\
i\left(t_{L}-t_{R}\right) \\
-i\left(t_{L}-t_{R}\right) / \eta_{0} \\
\left(t_{L}+t_{R}\right) / \eta_{0}
\end{array}\right)
$$

emerge from the electromagnetic field phasors at the entry and the exit planes, respectively. The $4 \times 4$ matrix

$$
\begin{array}{r}
\bar{M}=\bar{B}(h \pi / 2) \cdot\left[\bar{B}\left(h \frac{\pi L}{\Omega}\right) \cdot \exp \left(i \bar{A}^{\prime} L\right)\right] . \\
\bar{B}(-h \pi / 2) \cdot\left[\bar{B}\left(h \frac{\pi L}{\Omega}\right) \cdot \exp \left(i \bar{A}^{\prime} L\right)\right]
\end{array}
$$

captures the optical response characteristics of the centrally defective SCM slab, where

$$
\begin{gathered}
\bar{A}^{\prime}=\left(\begin{array}{cccc}
0 & -i \frac{h \pi}{\Omega} & 0 & \omega \mu_{o} \\
i \frac{h \pi}{\Omega} & 0 & -\omega \mu_{0} & 0 \\
-\omega \epsilon_{0} \epsilon_{E} & -\omega \epsilon_{o} \epsilon_{B} & 0 & -i \frac{h \pi}{\Omega} \\
\omega \epsilon_{0} \epsilon_{D} & \omega \epsilon_{o} \epsilon_{E} & i \frac{h \pi}{\Omega} & 0
\end{array}\right), \\
\bar{B}(\zeta)=\left(\begin{array}{cccc}
\cos \zeta & -\sin \zeta & 0 & 0 \\
\sin \zeta & \cos \zeta & 0 & 0 \\
0 & 0 & \cos \zeta & -\sin \zeta \\
0 & 0 & \sin \zeta & \cos \zeta
\end{array}\right), \\
\epsilon_{B}=\epsilon_{2}^{(0)}-\frac{\epsilon_{2}^{(0)}}{\epsilon_{1}^{(0)}} \epsilon_{m}, \\
\epsilon_{D}=\epsilon_{d}-\frac{\epsilon_{2}^{(0)}}{\epsilon_{1}^{(0)}}\left[\epsilon_{\iota} \cos \chi+\right. \\
\left(\epsilon_{j}+\epsilon_{\ell} \frac{\sin 2 \chi}{2}+\epsilon_{k} \sin \chi\right] \\
\epsilon_{E}=\frac{\epsilon_{2}^{(0)}}{\epsilon_{1}^{(0)}}\left(\epsilon_{e}+\epsilon_{h}\right)
\end{gathered}
$$

$$
\begin{gathered}
\epsilon_{d}=\frac{\epsilon_{1}^{(0)} \epsilon_{3}^{(0)}}{\epsilon_{1}^{(0)} \cos ^{2} \chi+\epsilon_{3}^{(0)} \sin ^{2} \chi} \\
\epsilon_{e}=E_{z}^{d c} \epsilon_{1}^{(0)} \epsilon_{d}\left(r_{41} \cos ^{2} \chi-r_{63} \sin ^{2} \chi\right), \\
\epsilon_{h}=E_{z}^{d c} \epsilon_{1}^{(0)} \epsilon_{d} \sin \chi \cos \chi\left(r_{43}-r_{61}\right), \\
\epsilon_{l}=E_{z}^{d c} \frac{\epsilon_{1}^{(0)}}{\epsilon_{2}^{(0)}} \epsilon_{d}^{2}\left(r_{31} \cos ^{2} \chi-r_{53} \sin ^{2} \chi\right), \\
\epsilon_{j}=E_{z}^{d c} \frac{\epsilon_{1}^{(0)}}{\epsilon_{2}^{(0)}} \epsilon_{d}^{2} \sin \chi\left(r_{11}-r_{53}\right), \\
\epsilon_{k}=E_{z}^{d c} \frac{\epsilon_{1}^{(0)}}{\epsilon_{2}^{(0)}} \epsilon_{d}^{2}\left(r_{13} \sin { }^{2} \chi-r_{51} \cos ^{2} \chi\right), \\
\epsilon_{\ell}=E_{z}^{d c} \frac{\epsilon_{1}^{(0)}}{\epsilon_{2}^{(0)}} \epsilon_{d}^{2} \cos \chi\left(r_{33}-r_{51}\right), \\
\epsilon_{m}=E_{z}^{d c} \epsilon_{1}^{(0)} \epsilon_{2}^{(0)}\left(r_{21} \cos \chi+r_{23} \sin \chi\right)
\end{gathered}
$$

The foregoing expression for $\bar{A}^{\prime}$ is correct to the first order in $E_{z}^{d c}$.

Parenthetically, although Eq.(3) is naturally independent of the direction of propagation of the incident plane wave, the matrix $\bar{A}^{\prime}$ becomes a function of $z$ for oblique-incidence conditions. However, the normal-incidence case appears to be the most application-oriented one, and therefore is focused upon this paper.

The reflection amplitudes $r_{L, R}$ and the transmission amplitudes $t_{L, R}$ can be determined for specified incident amplitudes ( $a_{L}$ and $a_{R}$ ) by solving Eq.(10) to yield:

$$
\begin{aligned}
& \left(\begin{array}{l}
r_{L} \\
r_{R}
\end{array}\right)=\left(\begin{array}{ll}
r_{L L} & r_{L R} \\
r_{R L} & r_{R R}
\end{array}\right)\left(\begin{array}{l}
a_{L} \\
a_{R}
\end{array}\right), \\
& \left(\begin{array}{c}
t_{L} \\
t_{R}
\end{array}\right)=\left(\begin{array}{ll}
t_{L L} & t_{L R} \\
t_{R L} & t_{R R}
\end{array}\right)\left(\begin{array}{l}
a_{L} \\
a_{R}
\end{array}\right) .
\end{aligned}
$$

Both $2 \times 2$ matrices in these equations are defined phenomenologically. The co-polarized transmission coefficients are denoted by $t_{L L}$ and $t_{R R}$, and the cross-polarized ones by $t_{L R}$ and $t_{R L}$; and similarly for the reflection coefficients in Eq.(27). Reflectances and transmittances are denoted, e.g., as $T_{L R}=\left|t_{L R}\right|^{2}$.

\subsection{Circular Bragg phenomenon}

For definiteness, let us set $h=1$, i.e., the chosen SCM slab is structurally right-handed. Suppose the central $90^{\circ}$-twist defect were to be absent. Then the matrices $\bar{B}( \pm h \pi / 2)$ would have to be removed from the right side of Eq.(13). With the twin assumptions of the absence of dispersion and the negligibly small dissipation, an incident RCP plane wave would be highly reflected and an incident LCP plane wave would be highly transmitted in the Bragg regime, provided the thickness ratio $L / \Omega$ were to be sufficiently high. 
By comparison to extant results for non-electro-optic SCMs [7], the center-wavelength of the Bragg regime for normal incidence is given by [27]

$$
\lambda_{0}^{B r}=\Omega\left(\sqrt{\epsilon_{B \zeta}}+\sqrt{\epsilon_{D \xi}}\right)
$$

and the corresponding full-width-at-half-maximum (FWHM) bandwidth by

$$
\left(\Delta \lambda_{o}\right)^{B r}=2 \Omega\left|\sqrt{\epsilon_{B \xi}}-\sqrt{\epsilon_{D \xi}}\right|,
$$

where

$$
\begin{aligned}
& \epsilon_{B \xi}=\frac{1}{2}\left[\epsilon_{B}+\epsilon_{D}+\sqrt{\left(\epsilon_{B}-\epsilon_{D}\right)^{2}+4 \epsilon_{E}^{2}}\right], \\
& \epsilon_{D \xi}=\frac{1}{2}\left[\epsilon_{B}+\epsilon_{D}-\sqrt{\left(\epsilon_{B}-\epsilon_{D}\right)^{2}+4 \epsilon_{E}^{2}}\right] .
\end{aligned}
$$

\section{N U MERICAL \\ RES U LT S \\ A N D DISCUSSION}

The central $90^{\circ}$-twist defect is responsible for the production of the two spectral holes centered at $\lambda_{0}^{B r}$, according to exact calculations [16]-[19], whereas the prediction of the approximate coupled-wave theory is very slightly different [17]. There are 20 classes of point group symmetry: cubic $23, \overline{4} 3 \mathrm{~m}$, tetragonal $4,4 \mathrm{~mm}, 422, \overline{4}, \overline{4} 2 \mathrm{~m}$, hexagonal $6,6 \mathrm{~mm}, 622, \overline{6}, \overline{6} \mathrm{~m} 2$, trigonal $3,3 m, 32$, orthogonal 222, $m m 2$, monoclinic $2, m$, and triclinic 1 . For each point group symmetry, $\lambda_{0}^{B r}$ was expanded in a three-term Maclaurin series with respect to $E_{z}^{d c}$. From the results, the 20 point group symmetries were classified into two groups:

- Group A: $\lambda_{0}^{B r}$ has zeroth-, first- and second-order dependences on $E_{z}^{d c}$ for tetragonal $4,4 \mathrm{~mm}, \overline{4}$, hexagonal 6, $6 m m, \overline{6}$, trigonal $3,3 m, 32$, orthogonal $m m 2$, and triclinic $1 ;$

- Group B: $\lambda_{0}^{B r}$ does not have the first- but has zerothand second-orders dependences on $E_{z}^{d c}$ for cubic 23, $\overline{4} 3 m$, tetragonal $422, \overline{4} 2 m$, hexagonal $622, \overline{6} m 2$, orthogonal 222, $m m 2$, monoclinic 2 , and $m$.

There ought to be pronounced differences between the responses of centrally defective SCM slabs with local point group symmetries belonging to Groups A and B, which indeed turned out to be true.

Figures 1 and 2 contain the spectrums of all four reflectances and all four transmittances, as functions of $E_{z}^{d c}$, of a SCM slab with local point group symmetry of the trigonal $3 m$ class and without a central defect. The relative permittivity scalars and the electro-optic coefficients used are those of lithium niobate [28], and $\Omega$ was chosen so as to position $\lambda_{0}^{B r}$ in the neighborhood of $650 \mathrm{~nm}$. The Bragg regime is evident through the elevated X-shaped feature in the plot of $R_{R R}$ and the absence of that feature in the plot of $R_{L L}$, in Figure 1. The transmittance plots in the next figure contain complementary signatures of the CBP as well, due to the satisfaction of the principle of conservation of energy [7] by all presented calculations.
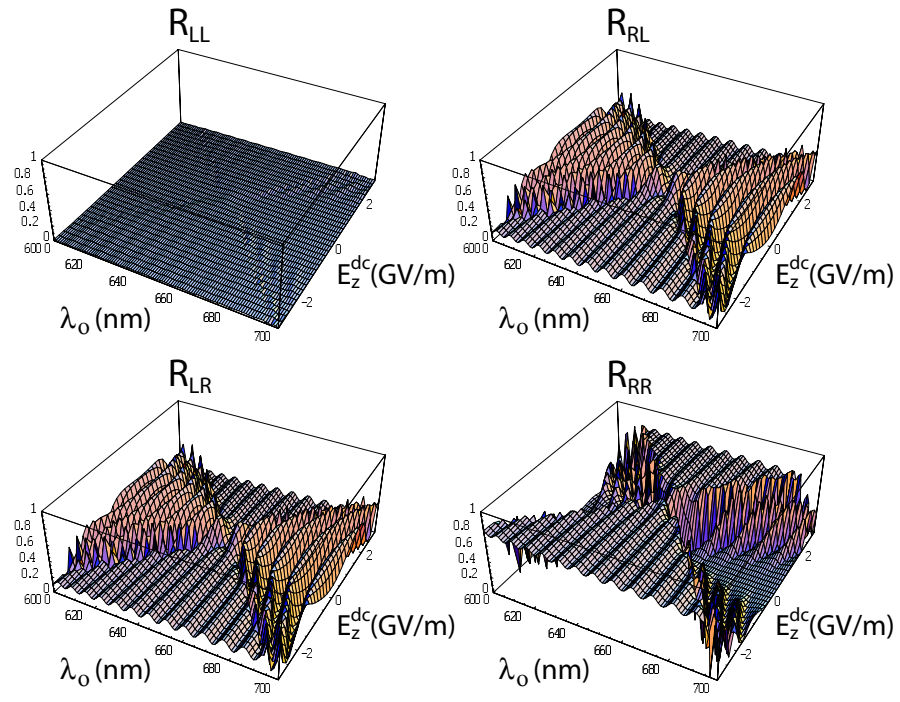

FIG. 1 Reflectances of a SCM slab of thickness $2 L$ as functions of the free-space wavelength $\lambda_{0}$ and the dc electric field $E_{z}^{d c}$. The SCM slab does not have a central defect. The local crystallographic class of the SCM is trigonal $3 m$. Other parameters are: $\epsilon_{1}^{(0)}=\epsilon_{2}^{(0)}=5.48, \epsilon_{3}^{(0)}=5.04, r_{22}=-r_{12}=-r_{61}=6.8 \times 10^{-12} \mathrm{~m} \mathrm{~V}^{-1}$, $r_{13}=r_{23}=9.6 \times 10^{-12} \mathrm{~m} \mathrm{~V}^{-1}, r_{33}=30.9 \times 10^{-12} \mathrm{~m} \mathrm{~V}^{-1}, r_{42}=r_{51}=$ $32.6 \times 10^{-12} \mathrm{~m} \mathrm{~V}^{-1}$, all other $r_{J K}=0, h=1, \Omega=140 \mathrm{~nm}, L=40 \Omega$, and $\chi=45^{\circ}$.
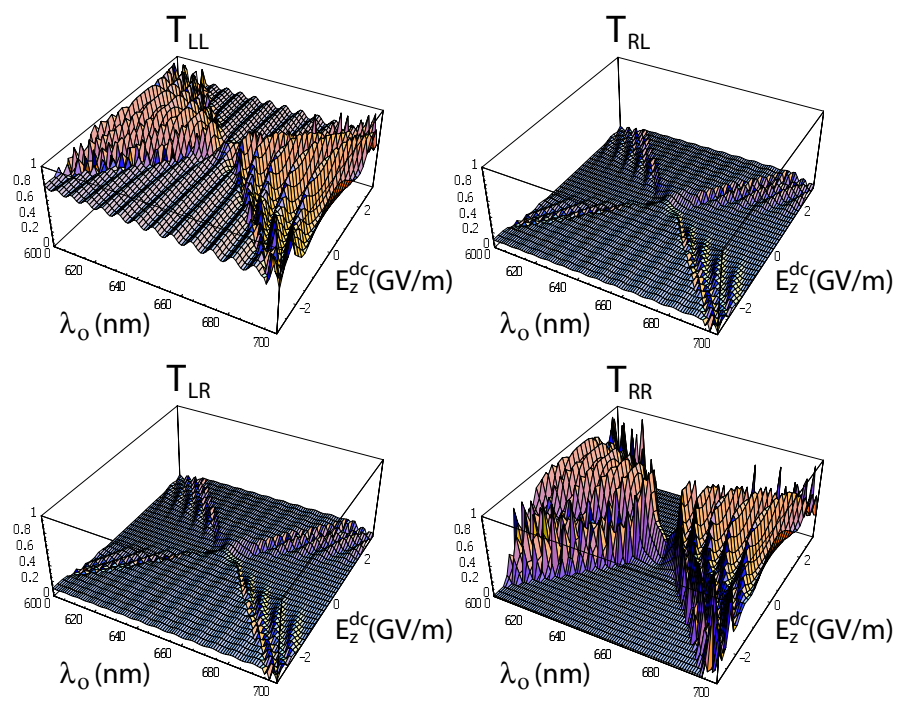

FIG. 2 Transmittances of a SCM slab of thickness $2 L$ as functions of the free-space wavelength $\lambda_{0}$ and the $d c$ electric field $E_{z}^{d c}$. The SCM slab does not have a central defect. Other parameters are the same as for Figure 1.

Close examination of Figures 1 and 2 reveals that the Bragg regime expands as $E_{z}^{d c}$ becomes more negative. However, as $E_{z}^{d c}$ increases positively from zero, the Bragg regime first contracts (as the CBP is not well-developed for $E_{z}^{d c} \sim$ $0.5 \mathrm{GV} \mathrm{m}^{-1}$ ) and then expands. This asymmetry with respect to the direction of the applied dc electric field is characteristic of Group A, and is engendered by the first-order dependence of $\lambda_{0}^{B r}$ on $E_{z}^{d c}$. Significantly, the plots indicate clearly that the Bragg regime can be manipulated with sufficiently large $\left|E_{z}^{d c}\right|$.

The introduction of a central $90^{\circ}$-twist defect gives rise to spectral holes. For moderate values of $\left|E_{z}^{d c}\right|$, a spectral hole appears in the spectrum of $R_{R R}$, but it wanes as $\left|E_{z}^{d c}\right|$ increases. 
This is evident in the somewhat magnified spectrums of $R_{R R}$ and $T_{R R}$ presented in Figure 3 for a SCM slab with a central defect. The plot of $R_{R R}$ clearly indicates a spectral hole for $E_{z}^{d c} \in[0,2] \mathrm{GV} \mathrm{m}^{-1}$, the FWHM bandwidth of the spectral hole being on the order of $1 \mathrm{~nm}$. Parenthetically, the broad ridges in these plots for $E_{z}^{d c} \sim 0.5 \mathrm{GV} \mathrm{m}^{-1}$ simply signify a poorly developed CBP.
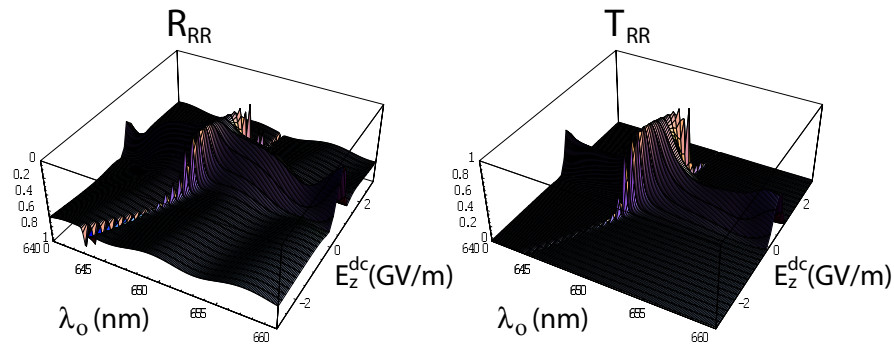

FIG. 3 Reflectance $R_{R R}$ and transmittance $T_{R R}$ of a SCM slab of thickness $2 L$ as functions of the free-space wavelength $\lambda_{0}$ and the dc electric field $E_{z}^{d c}$. The SCM slab has a central $90^{\circ}$-twist defect. Other parameters are the same as for Figure 1. The plot of $R_{R R}$ presents a spectral hole for roughly $E_{z}^{d c} \in[0,2] \mathrm{CV} \mathrm{m}^{-1}$. The fine structure evident in this figure as well as in the following three figures is due to the finite number of points chosen for the $\lambda_{0}$ and $E_{z}^{d c}$ axes.

As the first spectral hole (in the spectrum of $R_{R R}$ ) wanes with increasing $\left|E_{z}^{d c}\right|$, the second spectral hole (in the spectrum of $T_{L L}$ ) develops and matures. The FWHM bandwidth of this spectral hole is on the order of $0.1 \mathrm{~nm}$ and increases with $\left|E_{z}^{d c}\right|$, as the spectrums of $R_{L L}$ in Figure 4 show. Most importantly, the location of the second spectral hole varies smoothly with $E_{z}^{d c}$ for sufficiently high values of $\left|E_{z}^{d c}\right|$, blue shifting for negative $E_{z}^{d c}$ and red shifting for positive $E_{z}^{d c}$. Very precise tunability of the second spectral hole is indicated by the plots presented, and the feasibility of exploiting an electro-optic SCM slab with a central $90^{\circ}$-twist defect as an electrically tunable, $\mathrm{CP}$ rejection, narrow band filter is thereby established.
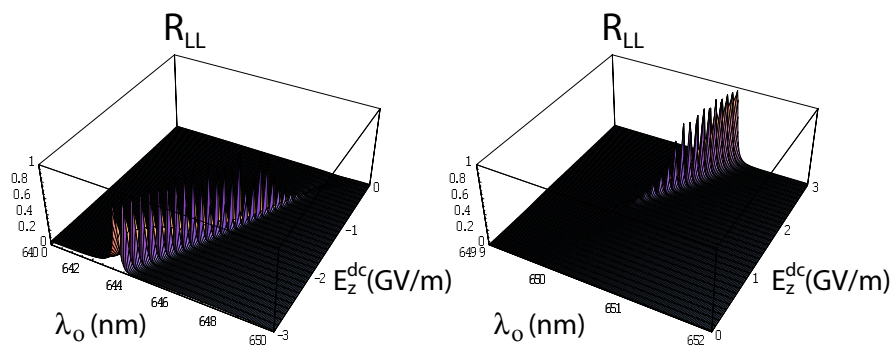

FIG. 4 Reflectance $R_{L L}$ of a SCM slab of thickness $2 L$ as a function of the free-space wavelength $\lambda_{0}$ and the dc electric field $E_{z}^{d c}$. The SCM slab has a central $90^{\circ}$-twist defect. Other parameters are the same as for Figure 1 .

Electrical control appears to require high dc voltages to be applied across SCM slabs, but the magnitudes of the dc voltages are comparable with the half-wave voltages of electro-optic materials, which are often in the $1-10 \mathrm{kV}$ range [28, 29]. Furthermore, the required magnitudes of $E_{z}^{d c}$ are much smaller than the characteristic atomic electric field strength [23]. The possibility of electric breakdown exists, but it would significantly depend on the time that the dc voltage would be switched on for.
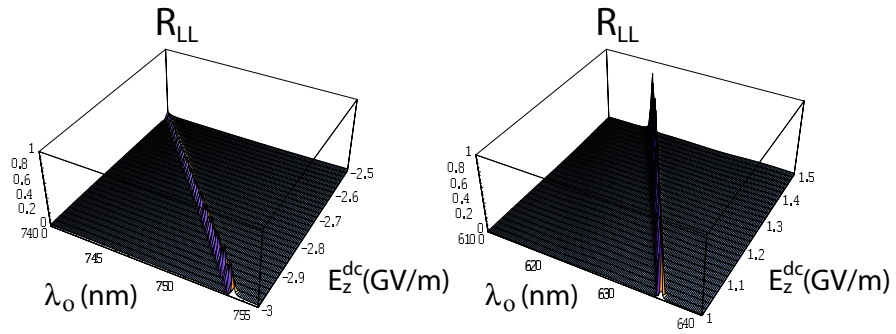

FIC. 5 Reflectance $R_{L L}$ of a SCM slab of thickness $2 L$ as a function of the free-space wavelength $\lambda_{0}$ and the dc electric field $E_{z}^{d c}$. The SCM slab has a central $90^{\circ}$-twist defect. The local crystallographic class of the SCM is orthorhombic mm2. Other parameters are: $\epsilon_{1}^{(0)}=4.72, \epsilon_{2}^{(0)}=5.20, \epsilon_{3}^{(0)}=5.43, r_{13}=34 \times 10^{-12} \mathrm{~m} \mathrm{~V}^{-1}$, $r_{23}=6 \times 10^{-12} \mathrm{~m} \mathrm{~V}^{-1}, r_{33}=63.4 \times 10^{-12} \mathrm{~m} \mathrm{~V}^{-1}, r_{42}=450 \times 10^{-12} \mathrm{~m} \mathrm{~V}^{-1}$, $r_{51}=120 \times 10^{-12} \mathrm{~m} \mathrm{~V}^{-1}$, all other $r_{J K}=0, h=1, \Omega=150 \mathrm{~nm}, L=24 \Omega$, and $\chi=90^{\circ}$

One way to reduce $\left|E_{z}^{d c}\right|$ is to use materials with larger electrooptic coefficients. Figure 5 contains spectrums of $R_{L L}$ computed with the relative permittivity scalars and the electrooptic coefficients of potassium niobate [28]. For positive as well as negative $E_{z}^{d c}$ of sufficiently large magnitude, a linear relationship between the dc electric field and the location of the second spectral hole is obvious. In particular, the dc electric field's magnitude is about half of that in Figure 4 when $E_{z}^{d c}>0$. Parenthetically, with increasing $\left|E_{z}^{d c}\right|$, the second spectral hole redshifts for negative $E_{z}^{d c}$ but blue shifts for positive $E_{z}^{d c}$, in contrast to Figure 4 .

All five figures presented thus far exemplify local point group symmetries in Group A. The plots of $R_{L L}$ in Figure 6 were computed with the permittivity scalars and the electro-optic coefficients of ammonium dihydrogen phosphate [28], which has a tetragonal $\overline{4} 2 \mathrm{~m}$ point group symmetry and thus belongs to Group B. Once again, the second spectral hole, captured in this figure, shifts with increasing $\left|E_{z}^{d c}\right|$; however, the shift is a blue shift regardless of the sign of $E_{z}^{d c}$. This insensitivity to the direction of the applied dc field is characteristic of SCM slabs with local point group symmetries in Group B.
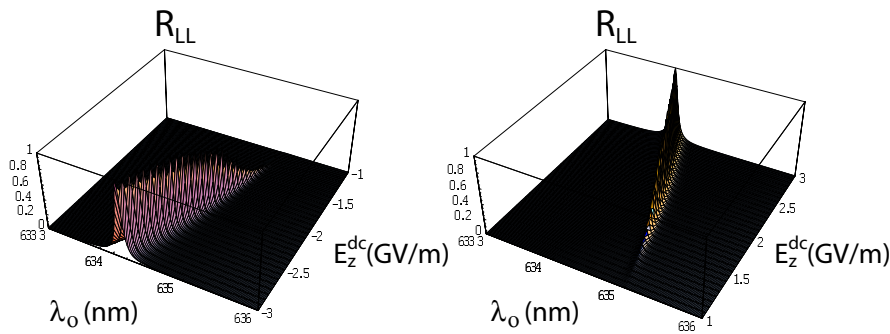

FIG. 6 Reflectance $R_{L L}$ of a SCM slab of thickness $2 L$ as a function of the free-space wavelength $\lambda_{0}$ and the dc electric field $E_{z}^{d c}$. The SCM slab has a central $90^{\circ}$-twist defect. The local crystallographic class of the SCM is tetragonal $\overline{4} 2 \mathrm{~m}$. Other parameters are: $\epsilon_{1}^{(0)}=\epsilon_{2}^{(0)}=2.34, \epsilon_{3}^{(0)}=2.20, r_{41}=r_{52}=24.5 \times 10^{-12} \mathrm{~m} \mathrm{~V}^{-1}$, $r_{63}=8.5 \times 10^{-12} \mathrm{~m} \mathrm{~V}^{-1}$, all other $r_{J K}=0, h=1, \Omega=210 \mathrm{~nm}, L=40 \Omega$, and $\chi=30^{\circ}$.

All other factors the same, cross-handed-transmittance spectral holes in centrally defective SCM slabs with local point 
group symmetries belonging to Group A will shift for moderate values of $E_{z}^{d c}$, whereas those in centrally defective SCM slabs with local point group symmetries belonging to Group B will shift for higher values of $E_{z}^{d c}$. Moreover, the spectral holes will either redshift or blue shift - depending on the sign of $E_{z}^{d c}$ and the relative strengths of the first- and second-order dependences of $\lambda_{0}^{B r}$ on $E_{z}^{d c}$ - for Group A, but the shifts will be insensitive to the sign of $E_{z}^{d c}$ for Group B. It is even possible for the Bragg regime to be greatly diminished and even eliminated, along with the spectral holes, for Group A because the first-order and the second-order dependences of $\lambda_{0}^{B r}$ on $E_{z}^{d c}$ could offset each other such that $\epsilon_{B \xi} \approx \epsilon_{D \xi}$ for physically relevant values of $E_{z}^{d c}$.

\section{CONCLUDING REMARKS}

The spectrum of the cross-handed transmittance of an electrooptic SCM slab that is helicoidally non homogeneous in the thickness direction and is endowed with a central $90^{\circ}$-twist defect, shows evidence of an ultranarrowband spectral hole when a sufficiently high dc electric field is applied between the entry and the exit planes. This spectral hole migrates on the wavelength axis as the applied dc electric field is altered, thereby establishing the use of a centrally defective SCM slab as an electrically tunable, circular-polarization rejection filter with ultra narrow bandwidth. The tunability may or may not depend on the direction of the applied dc electric field, depending on the local point group group symmetry of the chosen SCM. Materials with higher electro-optic coefficients are preferable, as lower dc electric fields have to be applied. The presented theoretical results are expected to encourage experimentalists to fabricate, characterize, and optimize the proposed device.

\section{References}

[1] W.H. Southwell, "Spectral response calculations of rugate filters using coupled-wave theory" J. Opt. Soc. Am. A 5, 1558-1564 (1988).

[2] H.A. Macleod, Thin-film optical filters (Institute of Physics, Bristol, United Kingdom, 2001).

[3] H.A. Haus and C.V. Shank, "Asymmetric taper of distributed feedback lasers" IEEE J. Quantum. Electron. 12, 532-539 (1976).

[4] C.P. Agrawal and S. Radic, "Phase-shifted fiber Bragg gratings and their application for wavelength demultiplexing" IEEE Photon Technol. Lett. 6, 995-997 (1994).

[5] S. Chandrasekhar, Liquid crystals (Cambridge University Press, Cambridge, United Kingdom, 1992).

[6] P.G. de Gennes and J. Prost, The physics of liquid crystals (Clarendon Press, Oxford, United Kingdom, 1993).

[7] A. Lakhtakia and R. Messier, Sculptured thin films: Nanoengineered morphology and optics (SPIE Press, Bellingham, WA, USA, 2005).

[8] Y.-C. Yang, C.-S. Kee, J.-E. Kim, H.Y. Park, J.-C. Lee and Y.-J. Jeon, "Photonic defect modes of cholesteric liquid crystals" Phys. Rev. E 60, 6852-6854 (1999).

[9] A. Lakhtakia and M. McCall, "Sculptured thin films as ultranarrowbandpass circular-polarization filters" Opt. Commun. 168, 457-465 (1999).
[10] I.J. Hodgkinson, Q.H. Wu, K.E. Thorn, A. Lakhtakia and M.W. McCall, "Spacerless circular-polarization spectral-hole filters using chiral sculptured thin films: theory and experiment" Op.t Commun. 184, 57-66 (2000).

[11] A. Lakhtakia, M.W. McCall, J.A. Sherwin, Q.H. Wu and I.J. Hodgkinson, "Sculptured-thin-film spectral holes for optical sensing of fluids" Opt. Commun. 194, 33-46 (2001).

[12] V.I. Kopp and A.Z. Genack, "Twist defect in chiral photonic structures" Phys. Rev. Lett. 89, 033901 (2002). [See also the correspondence on this paper: (i) C. Oldano, Phys Rev Lett. 91259401 (2003); (ii) V.I. Kopp and A.Z. Genack, Phys Rev Lett. 91, 259402 (2003).]

[13] F. Wang and A. Lakhtakia, "Specular and nonspecular, thicknessdependent spectral holes in a slanted chiral sculptured thin film with a central twist defect" Opt. Commun. 215, 79-92 (2003).

[14] I.J. Hodgkinson, Q.h. Wu, L. De Silva, M. Arnold, M.W. McCall and A. Lakhtakia, "Supermodes of chiral photonic filters with combined twist and layer defects" Phys. Rev. Lett. 91, 223903 (2003).

[15] M. Becchi, S. Ponti, J.A. Reyes and C. Oldano, "Defect mode in helical photonic crystals: An analytic approach" Phys. Rev. A 70, 033103 (2004).

[16] J. Schmidtke and W. Stille, "Photonic defect modes in cholesteric liquid crystal films" Eur. Phys. J. E. 12, 553-564 (2003).

[17] F. Wang and A. Lakhtakia, "Optical crossover phenomenon due to a central $90^{\circ}$-twist defect in a chiral sculptured thin film or chiral liquid crystal" Proc. R. Soc. Lond. A 461, 2985-3004 (2005).

[18] F. Wang and A. Lakhtakia, "Defect modes in multisection helical photonic crystals" Opt. Express 13, 7319-7335 (2005).

[19] C.G. Avendaño, S.Ponti, J.A. Reyes and C.Oldano, "Multiplet structure of the defect modes in $1 \mathrm{D}$ helical photonic crystals with twist defects" J. Phys. A: Math. Cen. 38, 8821-8840 (2005).

[20] J. Schmidtke, W. Stille and H. Finkelmann, "Defect mode emission of a dye-doped cholesteric polymer network" Phys. Rev. Lett. 90, 083902 (2003).

[21] J. Xu, A. Lakhtakia, J. Liou, A. Chen and I.J. Hodgkinson, "Circularly polarized fluorescence from light-emitting microcavities with sculptured-thin-film chiral reflectors", 0pt. Commun. accepted for publication (doi:10.1016/j.optcom.2006.02.025).

[22] A. Lakhtakia and J. Xu, "Planewave remittances of an axially excited chiral sculptured thin film with gain" Optik accepted for publication (doi:10.1016/j.ijle0.2006.01.011).

[23] R.W. Boyd, Nonlinear optics (Academic Press, San Diego, CA, USA, 1992).

[24] A. Lakhtakia, "Narrowband and ultranarrowband filters with electro-optic structurally chiral materials" Asian J. Phys. accepted for publication; also: http://www.arxiv.org/physics/0606031.

[25] I.J. Hodgkinson, Q.h. Wu, L. De Silva, M. Arnold, M.W. McCall and A. Lakhtakia, "Supermodes of chiral photonic filters with combined twist and layer defects", Phys. Rev. Lett. 91, 223903 (2003).

[26] F. Wang and A. Lakhtakia, "Third method for generation of spectral holes in chiral sculptured thin films" Opt. Commun. 250, 105-110 (2005).

[27] A. Lakhtakia, "Generalized Oseen transformation for and enhancement of Bragg characteristics of electro-optic structurally chiral materials" Opt. Commun. 261, 213-217 (2006).

[28] W.R. Cook Jr., Electrooptic coefficients, in: D.F. Nelson, LandoltBornstein Volume III/30A (Springer, Berlin, Germany, 1996).

[29] A. Yariv and P. Yeh, Photonics: Optical electronics in modern communications (Oxford University Press, New York, NY, USA, 2007). 